\title{
Quadratus Lumborum Block for Post-Operative Analgesia Following Anterior lliac Crest Bone Graft Harvesting.
}

\author{
Kumar Lokesh K S ${ }^{1}$, Rajalakshmi J \\ ${ }^{1}$ Associate professor, Anesthesiology, Sanjay Gandhi Institute of Trauma and Orthopedics, ${ }^{2}$ Consultant Anesthesiologist, Specialist Hospital. Department of \\ Anesthesiology, Specialist Hospital.
}

\section{Abstract}

Background: The anterior iliac crest is the most common site used for harvesting autologous bone graft. This can lead to acute and chronic iliac crest donor site pain leading to significant morbidity. Quadratus lumborum block is a newer advanced block which may help to alleviate this pain. This study aims to evaluate the effect of quadratus lumborum block on post operative analgesia in iliac crest bone graft patients. Subjects and Methods: A total of 60 patients aged between 18 yrs to 65 yrs were randomised into two equal groups. Group A received wound infiltration with local anesthesia. Group B received ultrasound guided quadratus lumborum block. Pain scores and opioid consumption were recorded in both groups. Results: Demographic profile was comparable in both the groups. The VAS score was significantly less in group B when compared to group A at 4th, 8th and 12th hrs ( $\mathrm{p}<0.0001$ ). The opioid consumption was $250.28 \pm 40.65$ in group A and $100.57 \pm 25.48$ group B. Conclusion: Ultrasound guided Quadratus lumborum block effectively reduces post operative pain in anterior iliac crest bone graft site when compared to local anesthetic wound infiltration.

Keywords: Quadratus lumborum block, anterior iliac crest graft, postoperative analgesia, thoracolumbar fascia.

Corresponding Author: Dr. Rajalakshmi J, 1025/2, 3rd cross, Sri kodandarama temple street, R S Palya, M S Nagar, Bangalore 560033.

Received: August 2019

Accepted: August 2019

\section{Introduction}

The Anterior iliac crest (AIC) is the most common site used for bone grafts as it is easily accessible, low morbidity and provides sufficient quantities of cortical and cancellous bone required for reconstructive surgeries. ${ }^{[1]}$ Most of the patients suffer from acute pain and almost 12 to $20 \%$ develop chronic pain leading to significant morbidity. ${ }^{[2,3]}$ Local anesthesia infiltration has been traditionally used to manage the post operative pain in such patients. ${ }^{[4]}$ However the effect of local anesthetic seemed to be variable and unreliable.

The iliac crest is supplied by the subcostal, ilioinguinal and iliohypogastric nerves. The lateral femoral cutaneous nerve supplies the skin overlying the antero lateral thigh and may contribute to the innervation. ${ }^{[5]}$ Though there are multiple ways to block these nerves, the quadratus lumborum block (QLB) which was recently introduced seems to be very effective in blocking these nerves and thereby provide optimal analgesia. ${ }^{[6]}$

Hence we conducted this study to evaluate the effectiveness of quadratus lumborum block in alleviating post operative pain at iliac crest bone graft site.

\section{Subjects and Methods}

This study was conducted at Sanjay Gandhi Institute of Trauma and Orthopedics and Specialist Hospital, Bangalore, after approval of the respective institutional ethical committee clearance. This study was conducted between Feb 2018 to July 2019. Written informed consent was obtained from all the patients. A total of 60 patients of ASA (American Society of Anesthesiologists) grade I and II between the age group of 18 to 65 years scheduled to undergo anterior iliac crest graft harvesting were included in the study. All the patients required anterior iliac crest graft for various surgeries involving the lower limbs like nonunion tibia, nonunion femur, ankle fusion etc.

Exclusion criteria included patients with history of cardiorespiratory disorders, hepatic and renal diseases, convulsions and neurological deficits, psychiatric diseases, ASA grade 3 and above. Patients were divided into 2 equal groups by computer generated random tables. Patients in both the groups received Subarachnoid block with Inj Hyperbaric Bupivacaine $0.5 \%$ as the primary anesthetic technique.

Group A patients received local wound infiltration with $0.5 \%$ bupivacaine just prior to incision (maximum $20 \mathrm{ml}$ ), administered by the operating surgeon. Group B patients received ultrasound guided quadratus lumborum block with $10 \mathrm{ml}$ of $2 \%$ lignocaine with adrenaline and $20 \mathrm{ml}$ of $0.5 \%$ Bupivacaine, administered by the anesthesiologist after subarachnoid block.

Technique

Patient was placed in lateral position with the side to be blocked placed superiorly. All aseptic precautions were 
followed. A low frequency curvilinear $2-5 \mathrm{Mhz}$ transducer probe was placed on the flank cranial to the anterior iliac crest. At the level of L3 transverse process the erector spinae muscle, psoas muscle, transverse abdominis muscle, internal oblique and external oblique muscle and quadratus lumborum muscle were identified. The quadratus lumborum muscle can be seen attaching to the tip of the transverse process of L3. $15 \mathrm{~cm} 22 \mathrm{G}$ insulated needle was inserted in plane in a lateral to medial direction with the needle tip advancing through the quadratus lumborum muscle and the anterior layer of the quadratus lumborum fascia. A volume of $10 \mathrm{ml}$ of $2 \%$ Lignocaine with adrenaline and $20 \mathrm{ml} 0.5 \%$ bupivacaine was administered in the fascial plane ventral to the quadratus lumborum muscle and posterolateral to psoas major muscle, while observing the spread under ultrasound. In the recovery room pain was assessed by the visual analogue scale. Pain score was evaluated at 1st, 4th, 8th, 12th, 18th and 24th hr. The opioid consumption and the time of administration of the first rescue analgesic was noted. Fentanyl was used as the rescue analgesic.

SPSS 22 program for microsoft windows was used for statistical analysis. All continuous data were expressed as meaan (SD) while the categorical data were expressed as percentages. Student $t$ test was used to compare the data in the two groups. A $p$ value of $<0.05$ was considered statistically significant.

\section{Results}

A total of 60 patients of either sex were divided into two equal groups. Patients demographic profile like age, sex and BMI was comparable in both the groups.

\section{Table 1: Demographics of the groups}

\begin{tabular}{|l|l|l|l|}
\hline Parameter & $\begin{array}{l}\text { Group A } \\
(\mathbf{n}=\mathbf{3 0})\end{array}$ & $\begin{array}{l}\text { Group B } \\
(\mathbf{n}=\mathbf{3 0})\end{array}$ & p-value \\
\hline Age $(\mathrm{yr})$ & $38.96 \pm 12.98$ & $38.76 \pm 12.07$ & 0.95 \\
\hline Male & $20(66)$ & $18(60)$ & 0.90 \\
\hline Female & $10(34)$ & $12(40)$ & 0.83 \\
\hline $\begin{array}{l}\text { Body mass } \\
\text { index }(\mathrm{kg} / \mathrm{m} 2)\end{array}$ & $26.34 \pm 3.54$ & $27.75 \pm 4.21$ & 0.16 \\
\hline
\end{tabular}

Table 2: Visual analogue scale (VAS), time to rescue analgesia and opioid consumption.

\begin{tabular}{|l|l|l|l|}
\hline & Group A & Group B & P value \\
\hline VAS at PACU & $0.82 \pm 0.15$ & $0.85 \pm 0.14$ & 0.42 \\
\hline VAS at 1st hr & $0.95 \pm 0.26$ & $0.98 \pm 0.18$ & 0.60 \\
\hline VAS at 4th hr & $2.45 \pm 1.13$ & $1.24 \pm 0.45$ & $<0.0001$ \\
\hline VAS at 8th hr & $3.96 \pm 0.99$ & $2.76 \pm 1.11$ & $<0.0001$ \\
\hline VAS at 12th hr & $3.85 \pm 1.05$ & $2.95 \pm 0.96$ & $<0.001$ \\
\hline VAS at 18th hr & $3.59 \pm 0.99$ & $3.25 \pm 1.69$ & 0.34 \\
\hline VAS at 24th hr & $3.88 \pm 1.23$ & $3.63 \pm 1.21$ & 0.43 \\
\hline $\begin{array}{l}\text { Time to rescue } \\
\text { analgesic }\end{array}$ & $5.35 \pm 1.76$ & $15.13 \pm 2.19$ & $<0.0001$ \\
\hline $\begin{array}{l}\text { Total opioid } \\
\text { consumption in } \\
\text { 24hrs }\end{array}$ & $250.28 \pm 40.65$ & $100.57 \pm 25.48$ & $<0.0001$ \\
\hline
\end{tabular}

VAS at PACU and at 1st hour was comparable in both the groups. VAS at 4th, 8th and 12th hour was low in quadratus lumborum group when compared to the control group. This reduction in VAS score was statistically very significant ( $\mathrm{p}$ value $<0.0001$ ). VAS at 18 th and 24th hour was comparable in both the groups. Patients in control group needed rescue analgesia much earlier when compared to patients who received quadratus lumborum block. Total opioid consumption (Fentanyl in mcg) was significanty lower in quadratus lumborum group (100) when compared to the control group (250).

\section{Discussion}

Our study demonstrated that patients who received quadratus lumborum block had significantly lower pain scores when compared to control group. The time required for rescue analgesia was longer in the quadratus lumborum group. The total opioid consumption was less in patients who received the block when compared to control group.

The iliac crest is considered the gold standard for autologous bone graft because of its large volume of cortical and cancellous bone. ${ }^{[7]}$ The periosteum of the iliac crest derives its rich innervation from the local nerves supplying the muscles attached to it. ${ }^{[5,8]}$ The outer lip of the AIC provides attachment to the iliotibial band, tensor fascia lata, and lower fibres of the external oblique as well as latissimus dorsi muscles. The intermediate part of the iliac crest allows attachment to the internal oblique muscle while the transversus abdominis muscle attaches to the inner lip of the anterior iliac crest. Hence the relevant innervation to the AIC is the lateral femoral cutaneous nerve of the thigh, ilioinguinal and iliohypogastric nerve, as well as the subcostal nerve.

The harvesting of anterior iliac crest bone graft leads to severe pain and sensory disturbances leading to significant morbidity. To overcome this pain there are various modalities of pain management which includes local anesthesia infiltration, ${ }^{[4]}$ local opioid infiltration, ${ }^{[9]}$ TAP block, ${ }^{[10]}$ paravertebral blocks and TFP block. ${ }^{[11]}$ However the analgesia provided by local anesthesia infiltration and opioids infiltration is often short lived and unreliable, often necessitating rescue medications. Although TAP (transversus abdominis plane) and TFP (transversalis fascia plane) blocks showed promising results, the spread and level of block achieved using a single injection was very variable and hence required multiple site injections for effective analgesia.

But the more recent quadratus lumborum block has shown promising results. ${ }^{[6]}$ Ultrasound guided quadratus lumborum block was first described by Blanco. ${ }^{[12]}$ It has been widely used as an analgesic technique for various surgeries like cesarean section, gynecological laparoscopic procedures, abdominal surgeries, appendectomy, gastrectomy, nephrectomy, hernioplasty, orchidopexy, cholecystectomy, hip and femur surgery. ${ }^{[13,14]}$

The quadratus lumborum, a small accessory muscle of inspiration takes its origin from the internal lip of the posterior segment of the iliac crest and the adjoining iliolumbar ligament. It gets inserted into the lower border of the twelfth rib and the tips of the transverse process of the upper four lumbar vertebrae. The quadratus lumborum and erector spinae muscles divide the thoraco lumbar fascia into three layers. The layer posterior to the erector spinae is known as the posterior thoracolumbar fascia, the layer intervening between the erector spinae and quadratus 
lumborum is known as the middle thoracolumbar fascia and the layer anterior to the quadratus lumborum is known as the transversalis fascia, which is in continuity with the transversalis fascia of the abdomen laterally, the psoas fascia medially and the fascia iliaca inferiorly. ${ }^{[15]}$

The mechanism of action of quadratus lumborum block is believed to be due to the spread of local anesthetics along the thoracolumbar fascia and the endothoracic fascia into the paravertebral space. Hence its assumed that visceral analgesia is due to the spread of anesthetics to the celiac ganglion or sympathetic trunk via splanchnic nerves similar to paravertebral block. The other mechanism of action is believed to be due to the thick network of sympathetic neurons in the superficial layer of thoracolumbar fascia. Blockade of pain receptors and mechanoreceptors present in the fascia also leads to analgesia. ${ }^{[13]}$ It is because of this ability of the quadratus lumborum block to provide visceral analgesia apart from somatic analgesia, its considered to be superior to local infiltration techniques and TAP blocks especially in various abdominal surgeries, open and laparoscopic.

There are 4 types of quadratus lumborum block, Type 1 to type 4 . In type 1 the drug is deposited in the lateral side of the quadratus lumborum muscle (Lateral QL block). In type 2 , the drug is deposited in the posterior side of the quadratus lumborum muscle (Posterior QL block). In type 3, the drug is deposited in the anterior portion of quadratus lumborum muscle posterior to the psoas major muscle (Anterior QL block). This approach assumes the shamrock sign, the transverse process of L4 vertebra is seen as a stem with erector spinae as posterior leaf, psoas major as anterior leaf and quadratus lumborum as the lateral leaf. ${ }^{[16]}$ The Anterior quadratus lumborum block includes transmuscular QL block, transverse oblique paramedian and the subcostal paramedial oblique sagittal TQL (transmuscular quadratus lumborum) block. ${ }^{[17]}$ Type 4 is intramuscular or depositing the local anesthetic into the quadratus lumborum muscle itself. ${ }^{[13]}$

Though quadratus lumborum block has been extensively used in the past few years for various surgeries as a mode of post operative analgesia, there is limited studies on its use in patients undergoing iliac crest bone grafting. Hence we conducted this study to evaluate the usefulness of quadratus lumborum block as post operative analgesic tool in patients undergoing anterior iliac crest bone graft harvesting.

Sondekoppam RV et al conducted a clinical and anatomical study on transmuscular quadratus lumborum block for analgesia following anterior iliac crest bone graft harvesting. ${ }^{[6]}$ In their study they placed a continuous transmuscular quadratus lumborum catheter and injected local anesthetic mixture, $20 \mathrm{ml}$ of $0.25 \%$ bupivacaine and $1 \%$ ropivacaine, 1:1 ratio. They noted the injectate spread corelated with the dermatomal anesthesia clinically and concluded that TQL may provide reliable analgesia for patients undergoing AIC bone graft harvesting.

Shabnam et al. ${ }^{[18]}$ reported a case series where they used quadratus lumboum block for iliac crest bone graft harvesting and found excellent post operative pain control. However they placed a contiuous infusion of $0.2 \%$ ropivacaine, $10 \mathrm{ml} / \mathrm{hr}$ through a catheter placed in the quadratus lumborum fascial plane.

Our study results correlated with the case series reports published by Sondekoppam RV et al and Shabnam et al. In our study we did a single shot quadratus lumborum block with $10 \mathrm{ml}$ of $2 \%$ Lignocaine with adrenaline and $20 \mathrm{ml}$ of $0.5 \%$ bupivacaine which provided effective analgesia upto $18 \mathrm{hrs}$ postoperatively. Therefore we conclude that quadratus lumborum block can provide efficient postoperative analgesia for anterior iliac crest bone graft harvest. The use of ultrasound improves the success rate of the block by allowing accurate localisation of target nerves and placement of the needle in precise position.

The limitations of our study is that we studied in a small group of patients. We did not assess the patient's pain scores after 24 hrs. Futher studies may be needed using large volume of patients and by comparing with other techniques like TAP, TFP and lumbar plexus block.

\section{Conclusion}

From our study we conclude that ultrasound guided quadratus lumborum block improves postoperative analgesia requirement in anterior iliac crest graft when compared to the control group.

\section{References}

1. Zenner J, Hitzl W, Mayer M, et al. Analysis of postoperative pain at the anterior iliac crest harvest site; a prospective study fo the intraoperative local administration of ropivacaine. Asian Spine J. 2015;9(1):39-46.

2. Kim DH, Rhim, R, Li L, et al. Prospective study of iliac crest bone graft harvest site pain and morbidity. Spine J. 2009;9(11):886-92

3. Armaghani SJ, Even JL, Zern EK, et al. The evaluation of donor site pain after harvest of tricortical anterior iliac crest bone graft for spinal surgery-: a prospective study. Spine(Philapa 1976). 2016;41(4):E191-6.

4. Brull SJ, Lieponis JV, Murphy MJ, et al. Acute and long term benefits of iliac crest donor site perfusion with local anesthetics. Anesth Analg 1992;74:145-7.

5. Birch R. chapter 80: Pelvic girdle, gluteal region and thigh. In: Standring, S, editor. Grays Anatomy: The anatomical basis of clinical practice. 41st ed. London: Elsevier; 2016.p. 1337-75.

6. Sondekoppam RV, Ip V, Johnston DF, et al. Ultrasound guided lateral medial transmuscular quadratus lumborum block for analgesia following anterior iliac crest bone graft harvesting; a clinical and anatomical study. Can J Anaesth. 2018; 65(2): 178-187.

7. Giannoudis PV, Karadimas EJ, Kanakaris NK. Anterior iliac crest bone graft harvesting. In:Giannoudis PV, editor. Practical procedures in orthopedic surgery. London: Springer Inc,;2012.p.51-5.

8. Howard MA, Dickie SR. Chapter 9: Comprehensive trunk anatomy. In: Neligan PC, editor. Plastic Surgery. 3rd ed. NY: Elsevier Health;2013. p.220-38

9. Reuben SS, Vieira P, Faruqi S, et al. Local administration of morphine for analgesia after iliac bone graft harvest. Anesthesiology 2001; 95:30-

10. Chiono J, Bernard N, Bringuier S, et al. The ultrasound guided transversus abdominis plane block for anterior iliac crest bone graft postoperative pain relief - a prospective descriptive study. Reg Anesth Pain Med 2010;35:520-4

11. Chin KJ, Chan V, Hebbard P, et al. Ultrasound guided transversalis fascia plane block provides analgesia for anterior iliac crest bone graft harvesting. Can J Anesth 2012;59:122-23

12. Blanco R. Optimal point of injection:the quadratus lumborum type I and II blocks. Anaesthesia 2013;68:4

13. Akerman M, Pejcic N and Velickovic. A review of the quadratus lumborum block and ERAS. Front. Med. 2018;5:44-51.

14. Chakraborty A, Khemka R, Datta T. Ultrasound guided truncal blocks: a new frontier in regional anesthesia. Indian J Anesth 2016;60:703-11.

15. Willard FH, Vleeming A, Schuenke MD et al. The thoracolumbar fascia: anatomy, function and clinical considerations. J Anat 2012; 221:507-36

16. Borglum J, Moriggl B, Jensen K, et al. Ultrasound guided transmuscular quadratus lumborum blockade. Br J Anaesth 2013;110:3

17. Elsharkawy H. Quadratus lumborum blocks. Advances in Anesthesia 
2017;35:145-57.

18. Majidian S, Jha S, Boyd E, et all. Ultrasound guided quadratus lumborum block for iliac crest bone graft harvesting. Paper presentation. 42nd Annual regional anesthesiology and acute pain medicine meeting. San Francisco, California. Apr 2017.

Copyright: (c) the author(s), publisher. Academia Anesthesiologica International is an Official Publication of "Society for Health Care \& Research Development". It is an open-access article distributed under the terms of the Creative Commons Attribution Non-Commercial License, which permits unrestricted non-commercial use, distribution, and reproduction in any medium, provided the original work is properly cited.

How to cite this article: Lokesh KSK, Rajalakshmi J. Quadratus Lumborum Block for Post-Operative Analgesia Following Anterior Iliac Crest Bone Graft Harvesting. Acad. Anesthesiol. Int. 2019;4(2):201-204.

DOI: dx.doi.org/10.21276/aan.2019.4.2.46

Source of Support: Nil, Conflict of Interest: None declared. 